\title{
Endovascular treatment of CCSVI in patients with multiple sclerosis: clinical outcome of 462 cases
}

\author{
A. Ghezzi - P. Annovazzi - E. Cocco • G. Coarelli • A. Lugaresi - M. Rovaris - F. Patti - E. Capello • \\ M. E. Rodegher $\cdot$ L. Moiola $\cdot$ S. Malucchi $\cdot$ G. Salemi $\cdot$ N. De Rossi $\cdot$ L. Provinciali $\cdot$ P. Perini $\cdot$ \\ R. Bergamaschi - E. Scarpini - G. Lus • A. Gallo $\cdot$ M. R. Tola • M. P. Amato • M. R. Rottoli \\ A. Bianchi - G. Comi - The MS Study Group-Italian Society of Neurology
}

Received: 25 September 2012/ Accepted: 7 January 2013

(C) Springer-Verlag Italia 2013

\begin{abstract}
Although it is still debated whether chronic cerebro-spinal venous insufficiency (CCSVI) plays a role in multiple sclerosis (MS) development, many patients underwent endovascular treatment (ET) of CCSVI. The objective of the study is to evaluate the outcome and safety of ET in Italian MS patients. Italian MS centers that are part of the Italian MS Study Group were all invited to participate to this retrospective study. A structured questionnaire was used to collect detailed clinical data before
\end{abstract}

Electronic supplementary material The online version of this article (doi:10.1007/s10072-013-1300-5) contains supplementary material, which is available to authorized users.

A. Ghezzi $(\bowtie) \cdot$ P. Annovazzi · A. Bianchi

Centro Studi Sclerosi Multipla, Ospedale di Gallarate,

Gallarate, Italy

e-mail: angelo.ghezzi@aogallarate.it

E. Cocco

Centro Sclerosi Multipla, Dipartimento di Sanità Pubblica,

Medicina Clinica e Molecolare, University of Cagliari,

Cagliari, Italy

G. Coarelli

Centre for Experimental Neurological Therapies (CENTERS),

Neurology and Department of Neurosciences, Mental Health

and Sensory Organs, Sapienza University S. Andrea

Hospital-Rome, Rome, Italy

A. Lugaresi

Multiple Sclerosis Center, Department of Neuroscience

and Imaging, Università "G d'Annunzio" Chieti, Chieti, Italy

M. Rovaris

Multiple Sclerosis Center, Fondazione Don Gnocchi,

Milan, Italy

F. Patti

DANA GF Ingrassia, Neuroscience Section, Multiple Sclerosis

Center, University of Catania, Catania, Italy and after the ET. Data from 462 patients were collected in 33 centers. ET consisted of balloon dilatation $(93 \%$ of cases) or stent application. The mean follow-up duration after ET was 31 weeks. Mean EDSS remained unchanged after ET (5.2 vs. 4.9), 144 relapses occurred in 98/462 cases $(21 \%)$, mainly in RR-MS patients. Fifteen severe adverse events were recorded in $3.2 \%$ of cases. Given the risk of severe adverse events and the lack of objective beneficial effects, our findings confirm that at present ET should not be recommended to patients with MS.

Keywords Multiple sclerosis · CCSVI $\cdot$ Endovascular treatment

\section{E. Capello}

Department of Neuroscience, Rehabilitation, Ophthalmology, Genetics, Maternal and Child Health, University of Genova, Liguria, Italy

M. E. Rodegher · L. Moiola · G. Comi

Department of Neurology, San Raffaele Scientific Institute,

Vita-Salute San Raffaele University, Milan, Italy

S. Malucchi

Neurologia 2-CRESM, AOU San Luigi Gonzaga,

Regione Gonzole 10, 10043 Orbassano, Turin, Italy

G. Salemi

Centro per la "Diagnosi e Cura delle Malattie Demielinizzanti"

Dipartimento di Biomedicina Sperimentale e Neuroscienze

Cliniche, Università degli Studi di Palermo, Palermo, Italy

N. De Rossi

U.S.D Neurologia, Centro Regionale per la Sclerosi Multipla, Spedali Civili Brescia, Presidio di Montichiari, Montichiari, Italy

L. Provinciali

Clinica Neurologica, Università Politecnica delle Marche,

Ancona, Italy 


\section{Introduction}

Chronic cerebro-spinal venous insufficiency (CCSVI) has been described as a condition of abnormal drainage of venous blood due to stenosis or malformation of the internal jugular and/or azygous veins $[1,2]$. Using the technique of transcranial color-coded duplex sonography this abnormality was detected by Zamboni et al. [3, 4] in $100 \%$ of patients with multiple sclerosis (MS): it was therefore claimed to play a relevant pathogenetic role in MS, causing an increased permeability of blood-brain barrier in perivenular regions, local iron deposition, and secondary multifocal inflammation.

No other study has confirmed the high frequency of abnormality reported by Zamboni et al. and a great variability of CCSVI prevalence has been found in MS patients, ranging from 0 to $92 \%$ [5, 6]. Moreover, this condition was also found in healthy subjects and in subjects with other neurological disorders [5]. The lack of reproducibility and specificity of the diagnostic procedures have been adduced as findings against a pathogenetic role of CCSVI in the development of MS [6,7].

Despite the unclear relationship between CCSVI and MS, many vascular surgeons or interventional radiologists have started to dilate neck veins by balloons or applying stents in MS patients, and many patients have decided to undergo such procedures. Some open-label studies have been published reporting a beneficial effect of endovascular treatment [8-15]. Nevertheless, side effects have also been reported [9, 13]. Furthermore, FDA has produced a document emphasizing the risks related to surgical interventions on neck veins [16].

In order to better evaluate the potential risks and benefits of CCSVI correction in MS, the present multicenter survey has been conducted in Italy to collect data from MS cases that underwent endovascular treatment for CCSVI.

\section{P. Perini}

Neurological Department, Azienda Ospedaliera Università' di Padova, 30128 Padua, Italy

R. Bergamaschi

Centro di Ricerca Interdipartimentale per la Sclerosi Multipla (CRISM), Istituto Neurologico Nazionale 'C. Mondino', Pavia, Italy

E. Scarpini

Clinica Neurologica, Fondazione Cà Granda Ospedale

Policlinico, Milan, Italy

G. Lus

First Division of Neurology, Department of Clinical and Experimental Medicine and Surgery, Second University of Naples, Naples, Italy

\section{Materials and methods}

Study design

This observational cohort study has been conducted within the network of Italian MS centers, coordinated by the MS Study Group of the Italian Society of Neurology. Thirtythree MS centers participated to the study. The study started in October 2011, after the design was presented during the Italian Congress of Neurology, and data collection was open until February 2012. It has been approved by the Ethical Committee of the coordinating center.

During the visit to MS centers, patients were regularly asked whether they underwent endovascular treatment for MS, unless they spontaneously declared it, and consecutively recruited. If the answer was positive, an ad hoc questionnaire was filled, anonymised, and sent to the coordinating center.

\section{Questionnaire}

A structured questionnaire was used to collect detailed demographic and clinical data, including data on neurological status and MRI (if available) before, after the intervention, and at the last available visit. Neurological status was scored using Kurtzke FS and EDSS scales [17]. Other items of the questionnaire included patient's subjective clinical outcome, adverse events, and previous and ongoing pharmacological treatments. On the whole, 28 items were included and analyzed.

\section{Results}

Data collected from 462 patients have been included in the database and have been analyzed. Demographic and clinical findings are summarized in Table 1. 
Endovascular CCSVI treatment consisted of balloon dilatation in $93 \%$ of cases and in stent application in $7 \%$.

The clinical outcome was assessed at a variable interval after the intervention: the mean follow-up duration was $30.7 \pm 36.1$ weeks (median 24 weeks, I quartile 12 weeks, III quartile 41 weeks). Results of EDSS score and of subjective evaluation in relation to the length of follow-up are reported in Table 2. The EDSS of the whole cohort at the last observation was $5.2 \pm 2.0$, with a mild but not significant increase compared to the baseline score (n.s. at paired at $t$ test).

Data have also been analyzed in the subgroup of 242 patients $(53 \%)$ who reported that ET improved their general-neurological condition: after a mean follow-up of $29.5 \pm 34.6$ weeks the final EDSS was $5.2 \pm 2.0$, starting from a mean basal score of $4.9 \pm 2.0$ (n.s. at paired $t$ test)

The distribution of cases according to EDSS scores, before and after the intervention, is reported in Fig. 1 (n.s. at Chi-square test). In the large majority of patients (281: $60.8 \%$ ) the final EDSS score was unchanged, it improved by 0.5 in $24(5.3 \%)$ cases, by 1 point or more in $20(4.4 \%)$ cases, it worsened by 0.5 in $76(16.4 \%)$ cases, and by 1 point or more in $61(13.1 \%)$ cases. No significant difference between pre- and post-ET EDSS score was found in relation to clinical course (RR/SP/PP), gender, age (cut-off was median age: 44 years), disability (cut-off was median EDSS: 4.5) and disease duration (cut-off was median disease duration: 12 years) (n.s. at Chi-square test).

One hundred and forty-four relapses occurred in 98/462 (21\%) patients (69 RR patients, 25 SP patients, and 4 PP patients): 58 relapses were observed in 26 out of 98 patients $(26 \%)$ who stopped the MS therapy after ET, and 50 relapses in 40 out of 189 patients $(21 \%)$ who continued the therapy ( $p=0.38$ ) (Table 3 ); among those who experienced a relapse, the mean number of relapses per patient was $1.4 \pm 0.7$ in the former and $1.2 \pm 0.5$ in the second group $(p=0.402)$.

One hundred and seventy-one subjects underwent brain MRI after the intervention (mean interval $28.8 \pm 37.4$ weeks).

Table 1 Demographic and clinical findings of MS patients who were submitted to endovascular treatment

\begin{tabular}{llllllr}
\hline & No. & F/M & Age (years) & Age at MS onset (years) & Disease duration (years) & $\begin{array}{l}\text { Treated with } \\
\text { immunomodulators/ } \\
\text { suppressants }\end{array}$ \\
\hline RR-MS & 222 & $137 / 85$ & $39.6 \pm 8.6$ & $27.0 \pm 15.3$ & $11.8 \pm 8.7$ & $169(76.1 \%)$ \\
SP-MS & 107 & $67 / 40$ & $46.5 \pm 9.0$ & $29.4 \pm 9.2$ & $16.6 \pm 6.1$ & $87(81.3 \%)$ \\
PP-MS & 133 & $74 / 59$ & $49.9 \pm 11.0$ & $36.9 \pm 11.2$ & $12.4 \pm 6.3$ & $31(23.3 \%)$ \\
Overall & 462 & $279 / 183$ & $40.0 \pm 10.0$ & $29.6 \pm 11.9$ & $13.6 \pm 7.0$ & $287(62.1 \%)$ \\
\hline
\end{tabular}

Table 2 Clinical outcome after ET in the whole cohort and in subgroups of patients according to the length of follow-up

\begin{tabular}{|c|c|c|c|c|c|c|}
\hline \multirow{2}{*}{$\begin{array}{l}\text { Follow-up duration } \\
\text { (months) }\end{array}$} & \multirow[t]{2}{*}{ No. of pts } & \multicolumn{2}{|l|}{ EDSS } & \multicolumn{3}{|c|}{ Subjective evaluation } \\
\hline & & Baseline & At follow-up & Improved $(\%)$ & Stable $(\%)$ & Worsened $(\%)$ \\
\hline$<3$ & 121 & $5.1 \pm 2.0$ & $5.2 \pm 2.0^{*}$ & 55.4 & 33.0 & 11.6 \\
\hline $3-6$ & 93 & $5.1 \pm 1.9$ & $5.2 \pm 2.0^{*}$ & 51.6 & 34.5 & 13.9 \\
\hline$>6$ & 248 & $4.8 \pm 2.1$ & $5.1 \pm 2.1^{*}$ & 48.3 & 42.0 & 9.7 \\
\hline Whole cohort & 462 & $4.9 \pm 2.0$ & $5.2 \pm 2.0^{*}$ & 52.5 & 36.5 & 11.0 \\
\hline
\end{tabular}

* n.s. at paired at $t$ test

Fig. 1 Distribution of patients (\%) according to EDSS $(<2,2-3.5,4-5.5,>5.5)$ before and after endovascular treatment (ET) (n.s. at Chi-square test) before ET



after ET

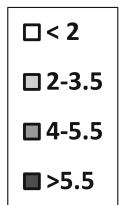

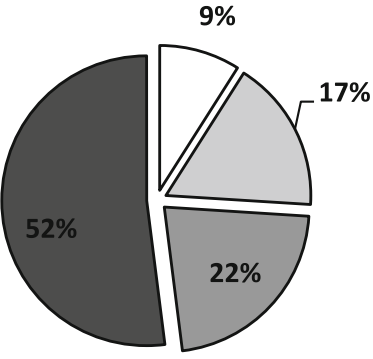

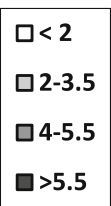


Table 3 Distribution of relapses after ET in relation to treatment for MS

\begin{tabular}{lrll}
\hline & No. & $\begin{array}{l}\text { Patients }(\%) \\
\text { with relapses }\end{array}$ & $\begin{array}{l}\text { No. of relapses } \\
\text { (relapses per patient) }\end{array}$ \\
\hline $\begin{array}{l}\text { Not treated } \\
\begin{array}{l}\text { Treated } \\
\text { Stopped after ET }\end{array}\end{array}$ & 98 & $26(172.3)$ & $36(1.1)$ \\
$\quad \begin{array}{l}\text { Continued after ET } \\
\text { 126.5) }\end{array}$ & $58(1.4)$ \\
\hline
\end{tabular}

Table 4 Severe adverse events occurring in MS patients who were submitted to endovascular treatment

\begin{tabular}{llll}
\hline$\#$ & Side effect & $\begin{array}{l}\text { Onset from } \\
\text { intervention }\end{array}$ & $\begin{array}{l}\text { EDSS } \\
\text { change }\end{array}$ \\
\hline 1 & Jugular thrombosis & $48 \mathrm{~h}$ & No \\
2 & Jugular thrombosis & 7 days & $2.5-3.5$ \\
3 & Jugular thrombosis & 30 days & No \\
4 & Jugular thrombosis & 30 days & No \\
5 & Jugular thrombosis & 6 weeks & No \\
6 & Jugular thrombosis & 10 weeks & $5-6$ \\
7 & Jugular thrombosis & 41 weeks & No \\
8 & Paroxysmal atrial fibrillation & 24 h & No \\
9 & Tetraventricular hydrocephalus & 48 h & $6-6.5$ \\
10 & Stroke & 12 weeks & $7.5-8.5$ \\
11 & Status epilepticus & 8 weeks & $4.5-9.5$ \\
12 & Aspiration pneumonia & 7 days & $7.5-9.0$ \\
13 & Hypertension and tachycardia & 30 days & No \\
& post sternotomy & & \\
14 & Severe bleeding from a bedsore & 15 days & No \\
15 & Myocardial infarction & 10 weeks & Death \\
\hline
\end{tabular}

Active lesions (i.e., contrast-enhancing or new T2-hyperintense) were observed in $61(35.7 \%)$ of these patients.

Adverse events were recorded in 50 patients $(13 \%)$ : they were mild in 35 patients $(7.5 \%)$ and mainly consisted of nausea, headache, cutaneous rash or other skin reactions, and inguinal haematoma.

Severe adverse events were recorded in 15 patients $(3.2 \%)$. They are summarized in Table 4 , and are more extensively described elsewhere [18].

\section{Discussion}

We are aware of the relevant limitations of this study, namely the observational design, the lack of an untreated control group, the lack of a blinded evaluation, the possible bias of patient selection. Nevertheless, our data describe the condition of MS patients who underwent endovascular treatment for CCSVI as observed in the "everyday clinical life" of Italian MS centers. Furthermore, to our knowledge this is the largest case record of MS patients treated for CCSVI ever published, and no randomized controlled trial is at present available.

We did not find a significant positive clinical outcome after ET in our patients. In the whole cohort as well as in the subgroup of "subjectively improved" patients the mean EDSS was unchanged at the last visit. Due to the unblinding of the observer this finding could not reliably reflect the real impact of endovascular procedure; however, using the occurrence of relapses as clinical end-point, that is a less subjective finding, $21 \%$ of the whole cohort developed a relapse in a relatively short interval after the intervention. The discontinuation of treatment for MS increased the risk of relapses, but they also occurred in patients without any previous treatment as well as in those who continued to take it. Moreover, many subjects presented an MRI reactivation of MS with the development of new or enhancing lesions.

More than $50 \%$ of cases of our cohort were affected by primary $(133,28.8 \%)$ or secondary progressive (107, $23.1 \%)$ MS. These patients frequently experience a negative evolution of the disease, without a positive effect of pharmacological treatments: due to media pressure, ET has been proposed, and perceived, as a possible therapeutic solution, generating many expectations. About $50 \%$ of cases of the whole cohort reported a subjective clinical benefit, but their EDSS remained unchanged after the treatment. The clinical outcome did not differ when data were analyzed in relation to clinical course, disease duration, gender, age.

To conclude, the results of our study do not show an outstanding beneficial effect of endovascular treatment in MS. Only a double-blind, placebo-controlled trial can give a definite answer about the actual impact of CCSVI endovascular treatment on MS evolution, once proven that this treatment is justified because of the unclear relationship between CCSVI and MS. A large multicenter study is in progress in Italy to evaluate the frequency of CCSVI in MS, healthy controls and other neurological degenerative disorders. On the other hand, the same methodological limitations we previously mentioned-mainly the lack of a control group and the unblinded evaluation-do characterize the studies that have reported positive results in terms of ET efficacy $[12,15,16]$.

The occurrence of adverse events, namely jugular thrombosis, in $2-55 \%$ of cases, more rarely vessel dissection, arrhythmias, vein breaking, stress-induced cardiomyopathy have been reported in previous studies [10, 11, 13]. FDA has recently pointed out the possible risks related to CCSVI endovascular treatment [16], having received reports of one patient who died from brain hemorrhage and another one who suffered permanent paralysis from a stroke after CCSVI 
treatment. Other reported serious complications of the CCSVI procedure including stent migration from the original location, venous injury, brain deep vein thrombosis, cranial nerves damage, and abdominal bleeding [16].

In our study, mild adverse events were observed in 35 patients; severe adverse events occurred in 15 patients after a variable interval from ET, including a patient who died because of myocardial infarction 10 weeks after ET: cases are more extensively presented in another paper [18].

To conclude, our results, observed in a large cohort of Italian MS patients, support the view that endovascular treatment can expose patients to severe, albeit relatively rare adverse events. Moreover, no clear beneficial clinical effect has been observed in our cohort after ET: the EDSS was unchanged after the procedure, relapses did not reduce, some patients presented new MRI lesions. Finally, the role of CCSVI has on MS pathogenesis has not been confirmed $[6,7]$ : the recent results of the largest cohort ever studied have shown that the prevalence of CCSVI is very low in MS patients, identical to that observed in normal subjects and in patients with other neurological disorders (Comi et al., paper in preparation). At present these findings confirm that CCSVI treatment should not be recommended to MS patients before additional studies will have better defined the real prevalence of CCSVI in MS and the benefit/risk profile of this treatment.

\section{References}

1. Zamboni P (2006) The big idea: iron-dependent inflammation in venous disease and proposed parallels in multiple sclerosis. J R Soc Med 99:589-593

2. Zamboni P, Menegatti E, Bartolomei I et al (2007) Intracranial venous haemodynamics in multiple sclerosis. Curr Neurovasc Res 4:252-258

3. Zamboni P, Galeotti R, Menegatti E et al (2009) Chronic cerebrospinal venous insufficiency in patients with multiple sclerosis. J Neurol Neurosurg Psychiatry 80:392-399
4. Zamboni P, Menegatti E, Galeotti R et al (2009) The value of cerebral Doppler venous haemodynamics in the assessment of multiple sclerosis. J Neurol Sci 282:21-27

5. Thapar A, Lane T, Nicholas R, Friede T et al (2011) Systematic review of sonographic chronic cerebrospinal venous insufficiency findings in multiple sclerosis. Phlebology 26(8):319-325

6. Baracchini C, Atzori M, Gallo P (2012) CCSVI and MS: no meaning, no fact. Neurol Sci May 9 (Epub ahead of print)

7. Bagert BA, Marder E, Stüve O (2011) Chronic cerebrospinal venous insufficiency and multiple sclerosis. Arch Neurol 68:1379-1384

8. Kipshidze N, Rukhadze I, Archvadze A et al (2011) Endovascular treatment of patients with chronic cerebrospinal venous insufficiency and multiple sclerosis. Georgian Med News 199:29-34

9. Mandato KD, Hegener PF, Siskin GP et al (2012) Safety of endovascular treatment of chronic cerebrospinal venous insufficiency: a report of 240 patients with multiple sclerosis. J Vasc Interv Radiol 23:55-59

10. Kostecki J, Zaniewski M, Ziaja K et al (2011) An endovascular treatment of chronic cerebro-spinal venous insufficiency in multiple sclerosis patients -6 month follow-up results. Neuro Endocrinol Lett 32:557-562

11. Zamboni P, Galeotti R, Weinstock-Guttman B et al (2012) Venous angioplasty in patients with multiple sclerosis: results of a pilot study. Eur J Vasc Endovasc Surg 43:116-122

12. Petrov I, Grozdinski L, Kaninski G (2011) Safety profile of endovascular treatment for chronic cerebrospinal venous insufficiency in patients with multiple sclerosis. J Endovasc Ther 18: 314-323

13. Ludyga T, Kazibudzki M, Simka M et al (2010) Endovascular treatment for chronic cerebrospinal venous insufficiency: is the procedure safe? Phlebology 25:286-295

14. Zamboni P, Galeotti R, Menegatti E et al (2009) A prospective open-label study of endovascular treatment of chronic cerebrospinal venous insufficiency. J Vasc Surg 50:1348-1358

15. Salvi F, Bartolomei I, Buccellato E et al (2012) Venous angioplasty in multiple sclerosis: neurological outcome at two years in a cohort of relapsing-remitting patients. Funct Neurol 27:55-59

16. http://www.fda.gov/MedicalDevices/Safety/AlertsandNotices/ucm 303318.htm\#.T6vm8Mc8cjc.email

17. Kurtzke JF (1983) Rating neurological impairment in multiple sclerosis: an expanded disability scale (EDSS). Neurology 33: $1444-1452$

18. Ghezzi A. Annovazzi P, Amato MP et al. (2013) Adverse events after endovascular treatment of chronic cerebrospinal venous insufficiency (CCSVI) in patients with multiple sclerosis Mult Scler $\mathbf{J}$ (in press) 